\title{
AN INEQUALITY FOR A LINEAR DISCRETE OPERATOR INVOLVING CONVEX FUNCTIONS
}

\section{ADRIAN HOLHOŞ}

Abstract. For the functional $A[f]=\sum_{k=1}^{m} a_{k} f\left(z_{k}\right)$, we give necessary and sufficient conditions over the real numbers $z_{k}$, such that, the inequality $A[f] \geqslant 0$, holds for some classes of convex functions. Then, we deduce an inequality related to Alzer's inequality and a weighted majorization inequality.

Mathematics subject classification (2000): Primary 05C38, 15A15; Secondary 05A15, 15A18. Keywords and phrases: linear discrete operator, convex functions, inequality, majorization.

\section{REFERENCES}

[1] S. Abramovich, J. Barić, M. Matić, J. PeČarić, On Van de Lune-Alzer's inequality, J. Math. Inequal., 1, 4 (2007), 563-587.

[2] I. GAVREA, Operators of Bernstein-Stancu type and the monotonicity of some sequences involving convex functions, International Series of Numerical Mathematics, Vol. 157, 181-192, Birkhäuser, 2008.

[3] J.-CH. KuAng, Some extensions and refinements of Minc-Sathre inequality, Math. Gaz., 83 (1999), $123-127$.

[4] T. Popoviciu, Notes sur les fonctions convexes d'ordre supérieur (III), Mathematica (Cluj), 16 (1940), 74-86.

[5] F. QI, Generalizations of Alzer's and Kuang's inequality, Tamkang J. Math., 31, 3 (2000), 223-227. RGMIA Res. Rep. Coll., 2, 6 (1999), Art. 12, 891-895. Available online at URL: http://www.staff.vu.edu.au/rgmia/v2n6.asp.

[6] F. QI, B.-N. GUO, Monotonicity of sequences involving convex function and sequence, Math. Inequal. Appl., 9, 2 (2006), 247-254. 